\title{
(6) OPEN ACCESS \\ Canakinumab treatment for patients with active recurrent or chronic TNF receptor-associated periodic syndrome (TRAPS): an open-label, phase II study
}

\author{
Marco Gattorno, ${ }_{1}^{1}$ Laura Obici, ${ }^{2}$ Marco Cattalini, ${ }^{3}$ Vincent Tormey, ${ }^{4}$ Ken Abrams, ${ }^{5}$ \\ Nicole Davis, ${ }^{5}$ Antonio Speziale, ${ }^{6}$ Suraj G Bhansali, ${ }^{5}$ Alberto Martini, ${ }^{1,7}$ \\ Helen J Lachmann ${ }^{8}$
}

\begin{abstract}
Handling editor Tore K Kvien
- Additional material is published online only. To view please visit the journal online (http://dx.doi.org/10.1136/ annrheumdis-2015-209031).

For numbered affiliations see end of article.

Correspondence to Dr Marco Gattorno, UO Pediatria 2, Istituto G. Gaslini, Via G. Gaslini 5, Genova 16147, Italy; marcogattorno@ ospedale-gaslini.ge.it
\end{abstract}

Received 15 December 2015 Revised 27 April 2016 Accepted 17 May 2016 Published Online First 7 June 2016
CrossMark

\section{SLinked}

http://dx.doi.org/10.1136 annrheumdis-2016-209335

\footnotetext{
To cite: Gattorno $M$, Obici L, Cattalini M, et al. Ann Rheum Dis

2017;76:173-178.
}

\section{ABSTRACT}

Objective To evaluate the efficacy of canakinumab, a high-affinity human monoclonal anti-interleukin-1 $\beta$ antibody, in inducing complete or almost complete responses in patients with active tumour necrosis factor receptor-associated periodic syndrome (TRAPS).

Methods Twenty patients (aged 7-78 years) with active recurrent or chronic TRAPS were treated with canakinumab $150 \mathrm{mg}$ every 4 weeks for 4 months ( $2 \mathrm{mg} / \mathrm{kg}$ for those $\leq 40 \mathrm{~kg}$ ) in this open-label, proof-ofconcept, phase II study. Canakinumab was then withdrawn for up to 5 months, with reintroduction on relapse, and 4 weekly administration (subsequently increased to every 8 weeks) for 24 months. The primary efficacy variable was the proportion of patients achieving complete or almost complete response at day 15 , defined as clinical remission (Physician's Global Assessment score $\leq 1$ ) and full or partial serological remission.

Results Nineteen patients (19/20, 95\%; 95\% Cl $75.1 \%$ to $99.9 \%$ ) achieved the primary efficacy variable. Responses to canakinumab occurred rapidly; median time to clinical remission 4 days ( $95 \% \mathrm{Cl} 3$ to 8 days). All patients relapsed after canakinumab was withdrawn; median time to relapse 91.5 days $(95 \% \mathrm{Cl} 65$ to 117 days). On reintroduction of canakinumab, clinical and serological responses were similar to those seen during the first phase, and were sustained throughout treatment. Canakinumab was well tolerated and clinical responses were accompanied by rapid and sustained improvement in health-related quality of life. Weight normalised pharmacokinetics of canakinumab, although limited, appeared to be consistent with historical canakinumab data.

Conclusions Canakinumab induces rapid disease control in patients with active TRAPS, and clinical benefits are sustained during long-term treatment. Trial registration number NCT01242813; Results.

\section{INTRODUCTION}

Tumour necrosis factor (TNF) receptor-associated periodic syndrome (TRAPS) is an autosomaldominant autoinflammatory disorder resulting from variants in the TNF super family receptor $1 \mathrm{~A}$ (TNFRSF1A) gene. ${ }^{1}$ Clinically, TRAPS is characterised by recurrent fever, myalgia, abdominal pain, rash, headaches, ocular symptoms and in some cases, amyloid A (AA) amyloidosis. ${ }^{2}{ }^{3}$ Diagnosis is challenging due to the considerable genetic heterogeneity and variable clinical presentation. ${ }^{4}$

Non-steroidal anti-inflammatory drugs (NSAIDs) can relieve fever but have little other benefit in TRAPS, and conventional immunomodulators are ineffective. ${ }^{2}$ Biologics that target specific proinflammatory cytokines have been investigated as treatment for TRAPS. At the time of the identification of the molecular defect, the TNF inhibitor etanercept was the cornerstone of TRAPS therapy ${ }^{1}$; it attenuates symptoms and inflammatory markers but many patients discontinue therapy due to perceived lack of efficacy. ${ }^{5-7}$ Paradoxically, treatment with the TNF inhibitors infliximab and adalimumab can precipitate inflammatory attacks and disease worsening. ${ }^{8-10}$

TNFRSF1A gene mutations in TRAPS are thought to cause aberrant trafficking and localisation of the type I TNF receptor, resulting in intracellular stress ultimately leading to increased production of interleukin-1 $\beta$ (IL-1 $\beta$ ). ${ }^{11}{ }^{12}$ While the pathogenesis of TRAPS is complex and still not completely understood, patients with TRAPS have excellent short-term response to the IL-1 receptor antagonist anakinra, suggesting IL- $1 \beta$ as a rational therapeutic target. $^{7} 1314$

Canakinumab is a high-affinity human monoclonal IL- $1 \beta$ antibody of the IgG $1 / \kappa$ isotype that binds to IL-1 $\beta$, thereby blocking the interaction of the cytokine with its receptor. ${ }^{15}{ }^{16}$ Case studies have demonstrated that patients with TRAPS can achieve a complete or near complete response with canakinumab treatment. ${ }^{17} 18$ This phase II proofof-concept study was designed to evaluate the efficacy and safety of canakinumab in inducing complete or almost complete responses within 15 days after the first dose in patients with active TRAPS.

\section{METHODS}

\section{Study design}

This open-label, single-treatment arm, proof-ofconcept, phase II study was conducted at six centres (four in Italy, one in England and one in Ireland) from 2010 to 2014. The study was registered at ClinicalTrials.gov (NCT01242813), and was conducted according to the ethical principles from the Declaration of Helsinki and in compliance with 
Good Clinical Practice. An independent ethics committee at each site approved the protocol.

The study enrolled patients with a clinical diagnosis of active recurrent or chronic TRAPS with a confirmed mutation of the TNFRSF1A gene (see online supplement for eligibility criteria), and consisted of a 4-month treatment period, followed by a withdrawal/follow-up period lasting up to 5 months, and, on disease relapse, a 24-month long-term treatment period (see online supplementary figure S1). Patients weighing $>40 \mathrm{~kg}$ received canakinumab $150 \mathrm{mg}$ subcutaneously once every four weeks during the treatment period (days 1, 29, 57 and 85). A single-dose up-titration to $300 \mathrm{mg}$ was permitted at day 8 in non-responders at the discretion of the treating physician. Patients weighing $\leq 40 \mathrm{~kg}$ received canakinumab $2 \mathrm{mg} / \mathrm{kg}$ once every four weeks, with a single-dose up-titration to $4 \mathrm{mg} / \mathrm{kg}$ allowed for non-responders at day 8. Patients who relapsed during the follow-up period received another dose of canakinumab equivalent to the last dose received, and then returned 2 weeks later for the end of follow-up visit. All patients who completed or relapsed during the follow-up period entered the long-term treatment period. In patients with a stable complete response, corticosteroid doses could be reduced at the investigators discretion from day 29 for complete responders only. The once every four weeks dosing regimen was chosen in the absence of sufficient clinical pharmacokinetic/pharmacodynamic data in patients with TRAPS. However, in the long-term treatment period, based on obtained pharmacokinetic/pharmacodynamic data, patients were transitioned to a dosing interval of once every eight weeks.

\section{Efficacy, pharmacokinetic and safety assessments}

Patients had visits on days 1 (baseline), 3, 8, 15, 29, 57 and 85, and then every 4 weeks during follow-up and long-term treatment. At each visit, investigators completed a global assessment using a 5-point Physician's Global Assessment (PGA) scale with scores of 0 (none), 1 (minimal), 2 (mild), 3 (moderate) and 4 (severe) for TRAPS-associated clinical signs and symptoms. The PGA was developed based upon discussions with the principal investigators and in accordance with the same instrument used previously in canakinumab trials in CAPS ${ }^{19}$ the PGA was not validated for use in TRAPS. Investigators also rated the severity of four-key associated signs and symptoms (rash, extremity musculoskeletal pain, abdominal pain and eye manifestations) using a 5 -point scale with scores of 0 (absent), 1 (minimal), 2 (mild), 3 (moderate) and 4 (severe). Fever $\left(>38.5^{\circ} \mathrm{C}\right.$ ) was also assessed at all visits. The inflammatory markers $\mathrm{C}$-reactive protein (CRP) and serum amyloid A (SAA) were measured at each visit; CRP levels were determined locally and standardised to a normal range of $0-10 \mathrm{mg} / \mathrm{L}$, and SAA levels were determined at the central laboratory.

The primary efficacy variable was the proportion of patients with active TRAPS achieving complete or almost complete response at day 15. Complete response was defined as clinical remission (PGA score $\leq 1$ ) with full serological remission (CRP $<10 \mathrm{mg} / \mathrm{L}$ and/or SAA $<10 \mathrm{mg} / \mathrm{L}$ ), and almost complete response was defined as clinical remission with partial serological remission $(\geq 70 \%$ reduction of baseline CRP and/or SAA). Non-response was defined as no change or worsening from baseline PGA score and/or increased or $<50 \%$ reduction from baseline CRP and/or SAA. Clinical relapse was defined by an increase in PGA score of $\geq 1$ point from day 15 to a PGA score $\geq 2$, and serological relapse was defined by an increase in CRP and/or SAA by $30 \%$ from day 15 to a value $\geq 30 \mathrm{mg} / \mathrm{L}$ unless it resulted from other factors (eg, concurrent infection).
Secondary efficacy variables included the proportion of patients with complete or almost complete response at day 8 , the proportion with clinical remission at days 8 and 15, the proportion with serological remission at days 8 and 15, the time to the investigator's assessment of clinical remission, and the time to relapse in the withdrawal phase. The designation of almost complete response was made to identify patients with evident amelioration of disease activity, and functionally served to select patients who did not need re-dosing with canakinumab.

Pharmacokinetic and safety assessments are described in the online supplement.

\section{Health-related quality of life}

Exploratory objectives included an assessment of long-term maintenance of disability and health-related quality of life (HRQoL) of canakinumab treatment in patients with TRAPS. Patients completed HRQoL questionnaires on days 1, 15, 113, 253 and then every 12 weeks during long-term treatment. Patients $\geq 18$ years of age at baseline completed the Medical Outcomes Study 36-Item Short Form Health Survey (SF-36), and those $<18$ years completed the Child Health Questionnaire (CHQ-PF50). Neither of these tools has been validated for use in TRAPS.

\section{RESULTS}

\section{Patients}

Twenty patients were treated with canakinumab and all entered the long-term treatment period. Eighteen patients completed the long-term treatment period. Two patients were lost to follow-up after receiving treatment to days 673 and 692, respectively. The mean age of the study cohort was 34.6 years; $95 \%$ were white and $65 \%$ were male (table 1 ). Six patients were $<18$ years of age (paediatric) and 14 patients were $\geq 18$ years of age (adult). The mean duration of time since TRAPS diagnosis at entry was 4.6 years. Nine patients had recurrent TRAPS, with a mean of $9.9 \pm 2.32$ episodes per year and episodes lasting for a mean of $11.9 \pm 3.76$ days. The remaining 11 patients had chronic TRAPS, with continuous symptoms or persistent elevation of acute phase reactants; one of those patients required continuous steroid treatment. Three patients (15\%) had AA amyloidosis, one of these also had a medical history of bronchiectasis and retroperitoneal fibrosis. Overall, 19 patients (95\%) received prior TRAPS treatment (table 1), which was discontinued per the protocol. Canakinumab was initiated when patients met criteria for active TRAPS but before they developed a severe disease flare.

\section{Efficacy}

The primary efficacy endpoint of complete or almost complete response at day 15 was achieved by 19 of 20 patients $(95 \%$; 95\% CI $75.1 \%$ to $99.9 \%$ ). The other patient had achieved a complete response at day 8 , but lost the serological response at day 15 despite ongoing clinical remission. Overall, 16 patients $(80 \%)$ achieved a complete response at day 8 , and 2 additional patients $(n=18 ; 90 \%)$ experienced complete clinical remission (ie, PGA score $\leq 1$ ) at day 8 despite not yet achieving a serological response (figure 1). All patients remained in clinical remission during the 4-month treatment period except for one patient who had a PGA assessment of mild TRAPS activity at the day 85 dosing visit. The median time to clinical remission was 4 days (95\% CI 3 to 8 days) (see online supplementary figure S2). Canakinumab improved each of the key signs and symptoms of TRAPS (see online supplementary table S1). The 


\section{Table 1 Baseline demographics and disease characteristics}

\begin{tabular}{ll}
\hline Characteristic & Study cohort \\
Age, years & \\
Mean (SD) & $34.6(18.36)$ \\
Range & $7.0-77.8$
\end{tabular}

Age range, years

\begin{tabular}{|c|c|}
\hline$<18$ & $6(30)$ \\
\hline$\geq 18$ & $14(70)$ \\
\hline \multicolumn{2}{|l|}{ Sex, n (\%) } \\
\hline Male & $13(65)$ \\
\hline Female & $7(35)$ \\
\hline \multicolumn{2}{|l|}{ Race, n (\%) } \\
\hline White & 19 (95) \\
\hline Asian & $1(5)$ \\
\hline \multicolumn{2}{|l|}{ Duration since TRAPS diagnosis, years } \\
\hline Mean (SD) & $4.6(3.57)$ \\
\hline Range & $0.01-11.7$ \\
\hline Mutation of TNFRSF1A gene, $\mathrm{n}(\%)$ & $20(100)$ \\
\hline T50M & $4(20)$ \\
\hline C55Y & $2(10)$ \\
\hline $\mathrm{C} 33 \mathrm{Y}$ & $2(10)$ \\
\hline $\mathrm{C} 72 \mathrm{~N}$ & $2(10)$ \\
\hline $\mathrm{C} 29 \mathrm{Y}$ & $1(5)$ \\
\hline C52Y & $1(5)$ \\
\hline D42del & $1(5)$ \\
\hline C30R & $1(5)$ \\
\hline T371 & $1(5)$ \\
\hline $\mathrm{F} 60 \mathrm{~L}$ & $1(5)$ \\
\hline Y38S & $1(5)$ \\
\hline C43G & $1(5)$ \\
\hline $\mathrm{C} 72 \mathrm{Y}$ & $1(5)$ \\
\hline C81Y & $1(5)$ \\
\hline \multicolumn{2}{|l|}{ Diagnosis of TRAPS, $\mathrm{n}(\%)$} \\
\hline Chronic & $11(55)$ \\
\hline Relapsing & $9(45)$ \\
\hline Number of episodes/year: mean (SD) & $9.9(2.32)$ \\
\hline Duration of each episode, days: mean (SD) & $11.9(3.76)$ \\
\hline CRP, mg/L: median (range) & $125(6-564)$ \\
\hline SAA, mg/L: median (range) & $198(16-2270)$ \\
\hline \multicolumn{2}{|l|}{ PGA of TRAPS activity, $n(\%)$} \\
\hline 2 (mild) & $13(65)$ \\
\hline 3 (moderate) & $6(30)$ \\
\hline 4 (severe) & $1(5)$ \\
\hline \multicolumn{2}{|c|}{ Disease-related medication taken before but stopped prior to study entry, ${ }^{*} \mathrm{n}(\%$} \\
\hline IL-1 inhibitor (anakinra) & $13(65) \dagger$ \\
\hline TNF inhibitor (etanercept) & $6(30) \ddagger$ \\
\hline Glucocorticoids & $10(50) \S$ \\
\hline NSAIDs & $3(15) ף$ \\
\hline
\end{tabular}

${ }^{*}$ As per study protocol.

$+C R, n=12 ; P R, n=1$.

$\neq C R, n=3 ; P R, n=3$.

$\S C R, n=6 ; P R, n=2 ;$ other, $n=2$ (treatment of TRAPS attack, prophylaxis).

IPR, $n=3$.

CR, complete response; CRP, C-reactive protein (standardised); IL, interleukin; NSAID, non-steroidal anti-inflammatory drug: PGA, Physician's Global Assessment; PR, partial response; SAA, serum amyloid A; TNF, tumour necrosis factor; TRAPS, TNF receptor-associated periodic syndrome.

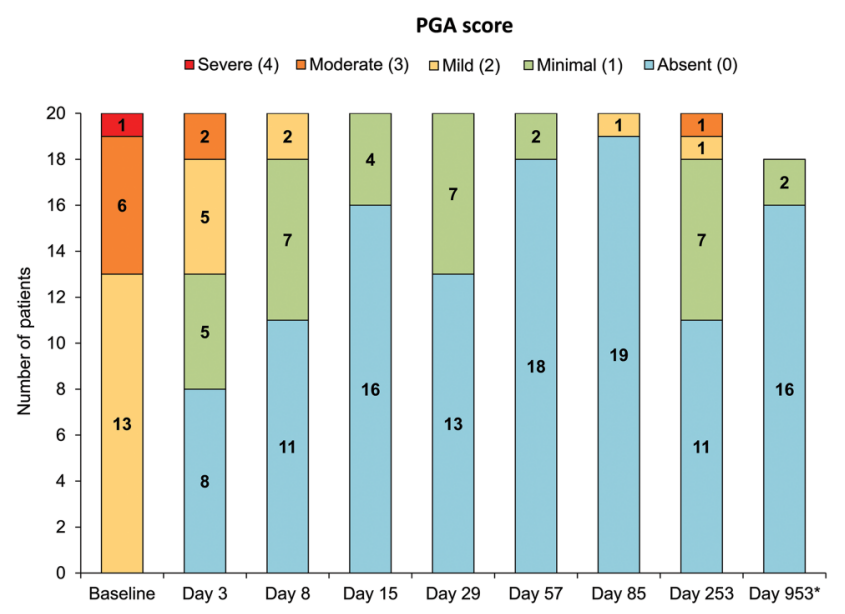

Figure 1 Distribution of Physician's Global Assessment (PGA) scores at various study visits. Clinical remission $=P G A \leq 1$. * Eighteen patients remained in the study until the end.

six paediatric patients responded similarly to the adults, with all achieving a complete or almost complete response at day 15 .

The inflammatory biomarkers CRP and SAA decreased rapidly after initiation of canakinumab treatment. Both CRP and SAA were within normal ranges $(\leq 10 \mathrm{mg} / \mathrm{L})$ in seven patients $(35 \%)$ on day 8 and in 12 patients $(60 \%)$ on day 15 . For the study cohort, median CRP and SAA declined to $\leq 10 \mathrm{mg} / \mathrm{L}$ by day 15 and remained within a normal range for the duration of the treatment period (figure 2). Median CRP and SAA values were 4.8 and $2.2 \mathrm{mg} / \mathrm{L}$, respectively, at the end of the treatment period, and remained within the normal range throughout the long-term treatment period (see online supplementary table S2). At the end of study visit, median CRP and SAA levels were 4.0 and $4.85 \mathrm{mg} / \mathrm{L}$, respectively.

Up-titration was allowed for patients who did not achieve a complete or almost complete response at day 8 . Two of the four non-responders at day 8 were up-titrated and achieved a complete or almost complete response by day 15 . Despite not receiving up-titration the other two patients still achieved a complete or almost complete response at day 15 .

All 20 patients relapsed (11 mild, 7 moderate and 2 severe) during the canakinumab withdrawal/follow-up period. Based on Kaplan-Meier estimates, the median time to relapse following the last canakinumab dose was 91.5 days (95\% CI 65 to 117 days) (see online supplementary figure S3). At 2 weeks after re-starting canakinumab following relapse, all patients returned to the level of response they had achieved prior to stopping canakinumab.

Based on analysis of time to relapse, the pharmacokinetic data obtained, and the desire of both patients and physicians to decrease the dose frequency, all patients in the long-term treatment period transitioned to a maintenance dosing interval of once every eight weeks of the last dose they received on day 85 (ie, 150 or $300 \mathrm{mg}$ (or 2 or $4 \mathrm{mg} / \mathrm{kg}$, respectively, if $<40 \mathrm{~kg}$ )). For seven patients (35\%; six adults, one child), the decreased dosing frequency at the lower 150-mg dose was not adequate to maintain disease control: three patients experienced one relapse episode, three patients experienced two relapse episodes and one patient experienced five relapse episodes. Four patients received NSAIDs and one patient received corticosteroid 


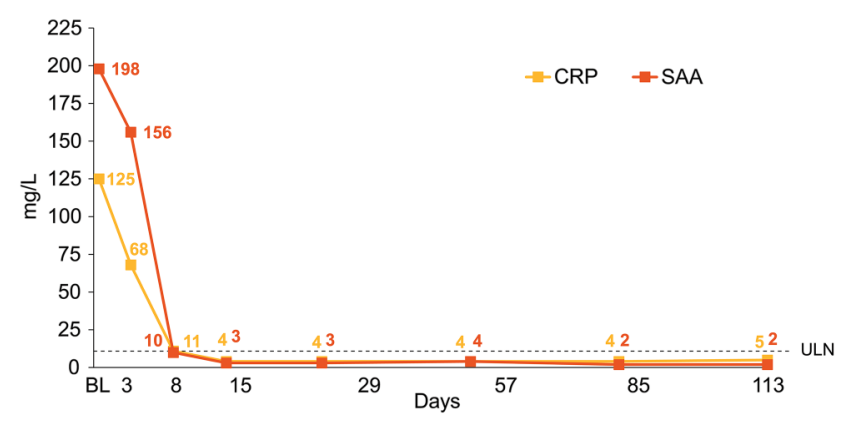

Figure 2 Median C-reactive protein (CRP) and serum amyloid A (SAA) values during the 4-month treatment period in the study cohort. $B L$, baseline; ULN, upper limit of normal.

treatment to manage these acute relapses. The dose of canakinumab was up-titrated to $300 \mathrm{mg}$ once every eight weeks in five of these patients, while the dose was not up-titrated in the remaining two patients, as they had isolated relapses not deemed severe or frequent enough by their treating physician to warrant a dose escalation.

\section{Health-related quality of life}

At baseline, 16 patients completed the SF-36 at least once. The patients exhibited considerable deficits in HRQoL compared with age and gender matched norms across all SF-36 domains; the mean differences on all domains exceeded the minimal clinically important difference of each scale and were statistically significant $(p<0.05)$. The greatest deficits in terms of effect sizes between patients with TRAPS and the general US population were observed for the role emotional, general health and bodily pain domains (figure $3 \mathrm{~A}$ ). When compared with benchmark samples of patients with other diseases, the HRQoL of the patients with TRAPS was most closely matched to patients with rheumatoid arthritis. Domain and summary SF-36 scores for the patients with TRAPS were consistently below those for patients with osteoarthritis or chronic back problems.

Treatment with canakinumab rapidly improved HRQoL in parallel with clinical improvement (figure 3B). Scores on all SF-36 domains increased from baseline to day $15(n=14)$, with mean scores $>50$ (general population average) on the physical functioning and vitality domains. By the end of treatment period on day $113(n=15)$, all domain scores were $>50$ except for general health and role emotional. The mean physical component summary score improved from 41.8 at baseline to 49.3 at day 15 and 51.4 at day 113; the mean mental component summary score showed improvements from 39.3 at baseline to 46.6 at day 15 and 49.0 at day 113 . For the five paediatric patients who completed the CHQ-PF50 questionnaire, the mean physical health and psychosocial scores at baseline were 35.4 and 52.7 , respectively. Improvements in mean physical health scores to $>40$ were evident by day 15 and were maintained at the end of treatment and end of follow-up visits. Mean psychosocial scores remained $>50$ throughout the study, except on days 365 and 617, they were noted at 46.6 and 47.2 , respectively.

\section{Pharmacokinetic profile}

In general, canakinumab displayed pharmacokinetic properties typical of an IgG1 monoclonal antibody. The pharmacokinetics of canakinumab in this population were consistent with that observed in other disease populations (see online supplementary table S3).

\section{Safety}

Canakinumab treatment was well tolerated; all patients reported at least one adverse event (AE), most commonly nasopharyngitis, abdominal pain, headache and oropharyngeal pain (table 2). Most AEs were mild to moderate in severity, and none led to study discontinuation. Serious adverse events, including pericarditis, abdominal pain, diarrhoea, intestinal obstruction, vomiting, upper respiratory tract infection, meniscus injury, hypertriglyceridaemia, hyperkalemia, pregnancy-related condition (ie, wife of enrolled patient became pregnant), foot deformity and condition aggravated occurred in seven patients. None of these events were suspected as drug-related study (see online supplementary table S4). There were no meaningful changes in haematology, clinical chemistry or vital signs during the study, and no neutralising anti-canakinumab antibodies were identified.

\section{DISCUSSION}

Canakinumab treatment produced rapid clinical and serological responses in paediatric and adult patients with active TRAPS. All patients achieved clinical remission in a median of 4 days after the first canakinumab dose. Importantly, both
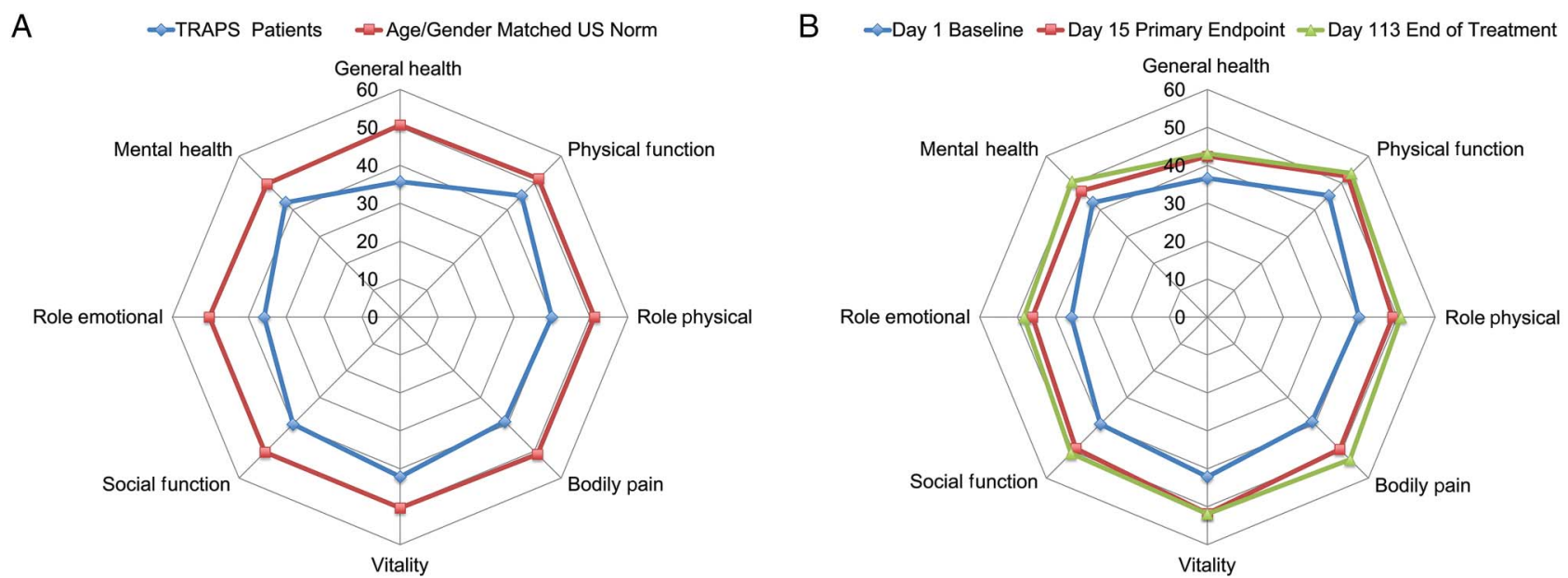

Figure 3 SF-36 spydergrams. (A) Comparison of tumour necrosis factor receptor-associated periodic syndrome (TRAPS) cohort with age and gender matched US population norm. (B) Effect of canakinumab at day 15 (primary endpoint) and day 113 (end of treatment period) compared with baseline. 
Table 2 Adverse events occurring in at least 3 patients by preferred term during the entire study (study duration: 33 months)

\begin{tabular}{ll}
\hline Adverse event, $\mathbf{n}$ (\%) & Study cohort $\mathbf{N}=\mathbf{2 0})$ \\
\hline Any adverse event & $20(100)$ \\
\hline Nasopharyngitis & $12(60)$ \\
\hline Abdominal pain & $11(55)$ \\
\hline Headache & $11(55)$ \\
\hline Oropharyngeal pain & $11(55)$ \\
\hline Fever & $10(50)$ \\
\hline Diarrhoea & $9(45)$ \\
\hline Arthralgia & $8(40)$ \\
\hline Vomiting & $8(40)$ \\
\hline Fatigue & $7(35)$ \\
\hline Upper respiratory tract infection & $7(35)$ \\
\hline Cough & $6(30)$ \\
\hline Musculoskeletal pain & $6(30)$ \\
\hline Myalgia & $6(30)$ \\
\hline Dizziness & $5(25)$ \\
\hline Rhinitis & $5(25)$ \\
\hline Abdominal pain upper & $4(20)$ \\
\hline Bronchitis & $4(20)$ \\
\hline Condition aggravated & $4(20)$ \\
\hline Pharyngitis & $4(20)$ \\
\hline Back pain & $3(15)$ \\
\hline Chest pain* & $3(15)$ \\
\hline Cystitis & $3(15)$ \\
\hline Ear pain & $3(15)$ \\
\hline Epistaxis & $3(15)$ \\
\hline Lower respiratory tract infection & $3(15)$ \\
\hline Pain in extremity & $3(15)$ \\
\hline Paraesthesia & $3(15)$ \\
\hline Rash & $3(15)$ \\
\hline Respiratory tract infection & $3(15)$ \\
\hline Toothache & $3(15)$ \\
\hline Viral infection & $3(15)$ \\
\hline
\end{tabular}

* Of these three events, two were non-cardiac chest pain and the other was related to tumour necrosis factor receptor-associated periodic syndrome (TRAPS) disease activity. None were considered related to canakinumab by the investigator.

median CRP and SAA declined into the normal range by day 15. The remarkable clinical and serological response was paralleled by a similarly rapid and robust improvement in HRQoL. Responses were sustained with continued canakinumab dosing; relapse occurred in all patients after withdrawal of canakinumab, but responses were restored after restarting canakinumab treatment.

This study corroborates findings from previous anecdotal reports in which the IL-1 receptor antagonists anakinra ${ }^{14}{ }^{20}$ and canakinumab $^{18}$ were used in the treatment of patients with refractory TRAPS, and support the key role of IL- $1 \beta$ rather than TNF in the pathogenesis of TRAPS. Clinically, TNF inhibition with etanercept, has proved disappointing in TRAPS. ${ }^{121}$ In the only prospective, open-label study, the benefit of etanercept was not sustained over time and 8 of 14 patients discontinued etanercept in the first 2 years, mainly due to lack of efficacy. ${ }^{5}$

Prior to our study, $65 \%$ of the patients had been treated successfully with the IL-1 receptor antagonist anakinra, and after a brief washout period, all responded to canakinumab, further supporting the long-term efficacy of IL-1 inhibition compared with TNF inhibition in patients with TRAPS. ${ }^{7}$

For the long-term period, patients were transitioned from canakinumab once every four weeks to once every eight weeks, the same dosing interval indicated for patients with another hereditary fever syndrome, cryopyrin-associated periodic syndromes. ${ }^{15}$ However, because maintenance canakinumab at $150 \mathrm{mg}$ once every eight weeks led to relapses in a significant minority $(35 \%)$ of patients, a 150 -mg once every four weeks regimen has been adopted for the ongoing pivotal phase III clinical trial (clinicaltrials.gov NCT02059291) evaluating efficacy and safety of canakinumab versus placebo in patients with hereditary periodic fever syndromes (TRAPS, familial Mediterranean fever and hyperimmunoglobulin D syndrome/ mevalonate kinase deficiency). ${ }^{22}$

At baseline, patients with TRAPS showed a clinically meaningful and statistically significant decrement in HRQoL compared with age and gender matched US population norms. Mean physical and mental component summary scores as well as 5 domain scores in the TRAPS cohort were 10 points below those for the US population (ie, 2 SDs lower). Notably, treatment with canakinumab rapidly improved HRQoL, with SF-36 component and domain scores approaching or reaching those for the normal US population.

In general, canakinumab displayed pharmacokinetic properties typical of an IgG1 monoclonal antibody with findings in this population consistent with that observed in other disease populations.

The benign safety profile observed in this small study was consistent with that observed in larger blinded trials of canakinumab in other conditions, including CAPS and systemic idiopathic juvenile arthritis. ${ }^{23}{ }^{24}$ The oldest patient treated in this study was 77 years old with comorbidities, and she has been treated successfully for several years without serious adverse events.

In summary, this study demonstrates that canakinumab produces rapid and robust clinical and serological responses, with parallel improvements in HRQoL, in patients with active TRAPS. This study supports continued exploration of the use of canakinumab in the treatment of TRAPS.

\section{Author affiliations}

${ }^{1}$ UO Pediatria 2, Reumatologia, Eurofever project, G Gaslini Institute, Genoa, Italy ${ }^{2}$ Amyloidosis Research and Treatment Centre, Biotechnology Research Laboratories, Fondazione IRCCS Policlinico San Matteo, Pavia, Italy

${ }^{3}$ Pediatric Clinic, University of Brescia and Spedali Civili, Brescia, Italy

${ }^{4}$ Galway University Hospitals, Galway, Ireland

${ }^{5}$ Novartis Pharmaceutical Corporation, East Hanover, New Jersey, USA

${ }^{6}$ Novartis Pharma AG, Basel, Switzerland

${ }^{7}$ Department of Neurosciences, Rehabilitation, Ophthalmology, Genetics, and Maternal-Fetal Medicine (DINOGMI), University of Genoa, Genoa, Italy

${ }^{8}$ Division of Medicine, National Amyloidosis Center, University College London Medical School, London, UK

Acknowledgements We thank Elise Hellinger and BioScience Communications for providing editorial support on behalf of Novartis Pharma AG. The authors would also like to thank Roberta Caorsi, Silvia Federici and Martina Finetti for their help in data collection and David Floch for sample analysis.

Funding This study was sponsored and funded by Novartis.

Competing interests KA: employee of Novartis Pharmaceuticals Corporation. SB: employee of Novartis Pharmaceuticals Corporation. MC: received speaker fees and served as a consultant for Novartis and SOBI. ND: employee of Novartis

Pharmaceuticals Corporation. MG: received speaker fees and served as a consultant for Novartis and SOBI, and has received unrestricted grants for the Eurofever Registry from Novartis and SOBI. HL: received speaker fees and served as a consultant for Novartis and SOBI. AM: received speaker fees and served as a consultant for Novartis. LO: received speaker fees and served as a consultant for Novartis. AS: employee of Novartis Pharma AG. 
Ethics approval The study was conducted according to the ethical principles of the Declaration of Helsinki. The study protocol and all amendments were reviewed by the independent ethics committee (IEC) or institutional review board (IRB) for each centre: Clinical Research Ethics Committee, Merlin Park Hospital, Galway, Ireland; National Research Ethics Service, Southampton \& South West Hampshire REC(B), Berkshire, UK; Comitato Di Bioetica Dell' IRCCS Istituto Giannina Gaslini Di Genova, Genova, Italy; Comitato Di Bioetica Della Fondazione IRCCS Policlinico San Matteo Di Pavia, Pavia, Italy; Comitato Etico Azienda ospedaliera Spedali Civili Di Brescia, Brescia, Italy; Comitato Etico Dell'ASL 1 Di Agrigento, Agrigento, Italy.

Provenance and peer review Not commissioned; externally peer reviewed.

Open Access This is an Open Access article distributed in accordance with the Creative Commons Attribution Non Commercial (CC BY-NC 4.0) license, which permits others to distribute, remix, adapt, build upon this work non-commercially, and license their derivative works on different terms, provided the original work is properly cited and the use is non-commercial. See: http://creativecommons.org/ licenses/by-nc/4.0/

\section{REFERENCES}

1 McDermott MF, Aksentijevich I, Galon J, et al. Germline mutations in the extracellular domains of the $55 \mathrm{kDa}$ TNF receptor, TNFR1, define a family of dominantly inherited autoinflammatory syndromes. Cell 1999;97:133-44.

2 Hull KM, Drewe E, Aksentijevich I, et al. The TNF receptor-associated periodic syndrome (TRAPS). Emerging concepts of an autoinflammatory disorder. Medicine 2002;81:349-68.

3 Lachmann HJ, Papa R, Gerhold K, et al. The phenotype of TNF receptor-associated autoinflammatory syndrome (TRAPS) at presentation: a series of 158 cases from the Eurofever/EUROTRAPS international registry. Ann Rheum Dis 2014;73:2160-7.

4 Toplak N, Frenkel J, Ozen S, et al. An international registry on autoinflammatory diseases: the Eurofever experience. Ann Rheum Dis 2012;71:1177-82.

5 Bulua AC, Mogul DB, Aksentijevich I, et al. Efficacy of etanercept in the tumor necrosis factor receptor-associated periodic syndrome: a prospective, open-label, dose escalation study. Arthritis Rheum 2012;64:908-13.

6 Quillinan N, Mannion G, Mohammad A, et al. Failure of sustained response to etanercept and refractoriness to anakinra in patients with T50M

TNF-receptor-associated periodic syndrome. Ann Rheum Dis 2011;70:1692-3.

7 Ter Haar N, Lachmann H, Özen S, et al. Treatment of autoinflammatory diseases: results from the Eurofever Registry and a literature review. Ann Rheum Dis 2013;72:678-85

8 Jacobelli $S$, André M, Alexandara JF, et al. Failure of anti-TNF therapy in TNF receptor 1-associated periodic syndrome (TRAPS). Rheumatology (Oxford) 2007;46:1212-13.
9 Drewe E, Powell RJ, McDermott EM. Comment on: failure of anti-TNF therapy in TNF receptor 1-associated periodic syndrome (TRAPS). Rheumatology (Oxford) 2007;46:1865-6.

10 Nedjai B, Hitman GA, Quillinan N, et al. Proinflammatory action of the anti-inflammatory drug infliximab in tumor necrosis factor receptor-associated periodic syndrome. Arthritis Rheum 2009;60:619-25.

11 Gattorno M, Martini A. Beyond the NLRP3 inflammasome. Autoinflammatory disorders reach adolescence. Arthritis Rheum 2013;65:1137-47.

12 Bachetti T, Chiesa S, Castagnola P, et al. Autophagy contributes to inflammation in patients with TNFR-associated periodic syndrome (TRAPS). Ann Rheum Dis 2013;72:1044-52.

13 Simon A, Bodar EJ, van der Hilst JCH, et al. Beneficial response to interleukin 1 receptor antagonist in TRAPS. Am J Med 2004;117:208-10.

14 Gattorno M, Pelagatti MA, Meini A, et al. Persistent efficacy of anakinra in patients with tumor necrosis factor-associated periodic syndrome. Arthritis Rheum 2008;58:1516-20.

15 Lachmann $\mathrm{HJ}$, Lowe $\mathrm{P}$, Felix SD, et al. In vivo regulation of interleukin $1 \beta$ in patients with cryopyrin-associated periodic syndromes. J Exp Med 2009;206:1029-36.

16 Dhimolea E. Canakinumab. mAbs 2010;2:3-13.

17 Brizi MG, Galeazzi M, Lucherini OM, et al. Successful treatment of tumor necrosis factor receptor-associated periodic syndrome with canakinumab. Ann Intern Med 2012;156:907-8.

18 Lopalco G, Rigante D, Vitale A, et al. Tumor necrosis factor receptor-associated periodic syndrome managed with the couple canakinumab-alendronate. Clin Rheumatol 2015;34:807-9.

19 Lachmann HJ, Kone-Paut I, Kuemmerle-Deschner JB, et al. Use of canakinumab in the cryopyrin-associated periodic syndrome. N Engl J Med 2009;360:2416-25.

20 Obici L, Meini A, Cattalini M, et al. Favourable and sustained response to anakinra in tumour necrosis factor receptor-associated periodic syndrome (TRAPS) with or without AA amyloidosis. Ann Rheum Dis 2011;70:1511-12.

21 Drewe E, McDermott EM, Powell PT, et al. Prospective study of anti-tumour necrosis factor receptor superfamily $1 \mathrm{~B}$ fusion protein, and case study of anti-tumour necrosis factor receptor superfamily 1 A fusion protein, in tumour necrosis factor receptor associated periodic syndrome (TRAPS): clinical and laboratory findings in a series of seven patients. Rheumatology (Oxford) 2003;42:235-9.

22 Study of Efficacy and Safety of Canakinumab in Patients With Hereditary Periodic Fevers. https://clinicaltrials.gov/ct2/show/NCT02059291. ClinicalTrials.gov Identifier: NCT02059291 (accessed 15 Apr 2016).

23 Church LD, McDermott MF. Canakinumab: a human anti-IL-1b monoclonal antibody for the treatment of cryopyrin-associated periodic syndromes. Expert Rev Clin Immunol 2010;6:831-41.

24 Ruperto $\mathrm{N}$, Brunner $\mathrm{HI}$, Quartier $\mathrm{P}$, et al. Two randomized trials of canakinumab in systemic juvenile idiopathic arthritis. N Engl J Med 2012;367:2396-406. 